\title{
Experiencias del proyecto Las Tres Horas y Cantos de Difuntos
}

\section{Un acercamiento a la ritualidad del pueblo afroserrano}

del Ecuador

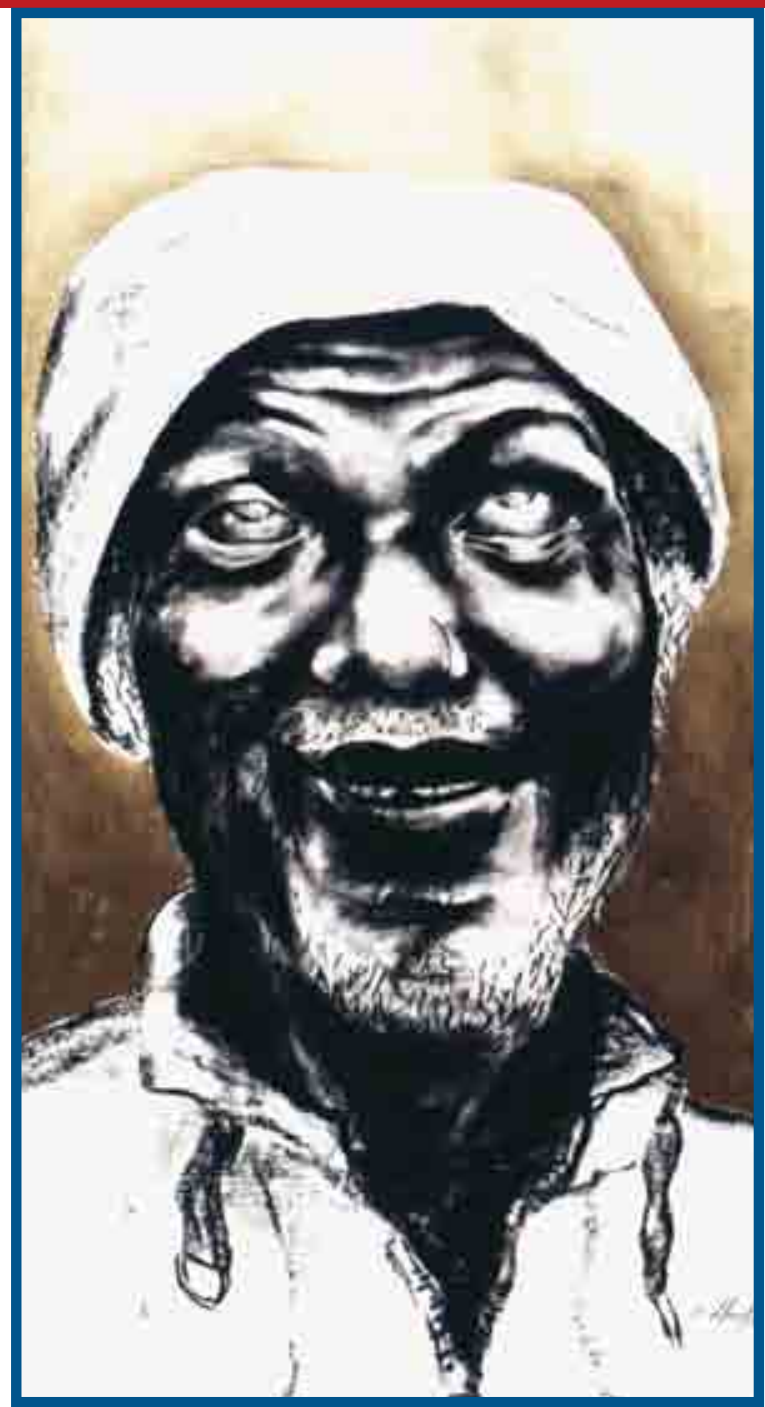

$S / T$

Mixta sobre papel, 2001

\section{Daniela Peña*}

\section{Palabras iniciales}

Recuerdo cuando estábamos sentadas con Las Tres Marías, esperando que esté listo el estudio para grabar su disco, y Doña Gloria de Chalguayacu nos dice: "qué lindo que sería grabar las Tres Horas, porque ya las jóvenes no quieren aprender" y se pusieron a cantar -creo que fue la primera palabra-.

A este momento le precede el nacimiento del Grupo Itinerante de Artes "Guandul" que -al igual que el frejolito- creció y se desarrolló en el valle del Chota, compartiendo la cotidianidad de la gente, aprendiendo y contagiándonos de su manera de sentir y pensar la vida y el mundo.

Cuando Las Tres Marías nos hablaron de lo lindo que sería grabar Las Tres Horas, se despertaron en mí varias cosas: por un lado, el gran cariño, gratitud y admiración por la gente del Chota, por otro, mi gusto por la música, por el canto, y por último, mi relación con la muerte.

Siento que estos tres elementos fueron los motores que impulsaron todo esto. 
Después de tener la idea latiendo en mí, se la pudo concretar a través de la carrera de Antropología Aplicada, con los fondos concursables que ofrecía la Universidad Politécnica Salesiana, como una forma de incentivar la investigación en los estudiantes.

En un inicio la idea fue grabar un $C D$ con el canto de Las Tres Horas de Semana Santa. Luego vi que los cantos de tiempos de finados y de los velorios eran igualmente importantes para la gente, así que grabamos primero con don Salomón Zura de Chalguayacu y con don Augusto Cribán de Caldera. Después escuché que había un grupo de cantoras bastante reconocido en la cuenca del río Mira, en la comunidad de La Concepción, así que hablé con doña Cremelia Chalá, la dirigente del grupo, y le pregunté si les gustaría participar en el disco. Finalmente, me comentaron que en Carcelén había también un grupo de cantoras provenientes de la comunidad de Santa Ana, en la cuenca del río Mira, que, pese a haber salido de su comunidad, no habían dejado esta tradición. De esta forma, se juntaron en un disco 31 cantoras de cuatro comunidades del valle del Chota y de la cuenca del río Mira.

Sabía que el $C D$ necesitaba de un acercamiento al contexto en donde se desarrollan los cantos. Es así que decidí escribir un pequeño folleto con muchas fotos que permitan acercarnos aún más al entorno y significado social de estos cantos.

Después me di cuenta que el folleto no iba a ser muy leído por la gente del Valle del Chota y de la cuenca del río Mira.

Recordaba también lo lindo que fue cuando en Chalguayacu proyectamos con "Guandul" algunos videos que se habían hecho en el valle del Chota y en la misma comunidad, hace varios años, uno de ellos hace más de veinte. Y me di cuenta que el verse a uno mismo, una misma, mueve procesos muy profundos en la construcción de la identidad.

Es así que, impulsada nuevamente por el carino y respeto que siento hacia la gente del Valle y de la cuenca del río Mira, fui descaradamente donde el vicerrector de la Universidad, para pedirle una ampliación del proyecto; es decir, para pedirle más dinero. Como es totalmente lógico, me dijo que primero termine el proyecto como lo había planteado inicialmente. Pero yo ya lo veía incompleto sin un trabajo audiovisual, así que, tercamente, seguí pidiendo apoyo a la universidad y busqué financiamiento por otros lados.

Por último, conversando con las cantoras residentes en Quito, con doña Maura Chalá, doña Chabela Chalá, veíamos lo lindo que sería hacer el lanzamiento del CD, como una forma de que la información no se quede en pocas manos sino que, más bien se socialicen las tradiciones del pueblo afroserrano.

Así que aquí estamos. Aquí están ellas y ellos para compartir con nosotros sus cantos, sus tradiciones, y aquí estamos nosotras, el Grupo Itinerante de Artes "Guandul”, para escuchar sus voces, aprender de ellas y crear espacios en donde otra gente, mucha gente, también escuche y aprenda de las voces del pueblo afroserrano.

Gracias.

Estas fueron las palabras iniciales en el evento del lanzamiento del libro- $C D$ y la presentación del video, el cual tuvo lugar en Quito el día 9 de abril de 2011 en el Teatro Variedades Ernesto Albán.

El propósito de presentar aquí este 'discurso' se debe a que existen varios elementos en estas palabras que quisiera subrayar como una forma de ir contando mis experiencias y conclusiones luego de haber realizado el proyecto de investigación y grabación.

\section{Compartiendo vivencias personales}

El proyecto inició en 2010, por la unión de dos necesidades: una proveniente de las cantoras de la comunidad de Chalguayacu, quienes preocupadas por la desaparición de los cantos 
quisieron impregnarlos en el tiempo y en el espacio a través de una grabación; y la otra, gracias a mi curiosidad como investigadora en los temas de las voces, las melodías, la música y, sobre todo, por el misterio que encierra la muerte; ésta se me presentó a los dieciséis años y pude enfrentarla justamente cantando. La necesidad manifestada por el grupo de Chalguayacu marcó una diferencia con el resto de cantoras, a quienes, si bien les gustó la idea, por no haber nacido de ellas, buscaban resultados de tipo económico, además que ya habían tenido una mala experiencia con alguna institución.

Mientras una se adentra más a la cultura, compartiendo, tanto los escenarios de acción (Turner, 1964) como la cotidianidad, entablando lazos vivenciales y afectivos, el proyecto se ve obligado a someterse a lo que dice Clifordd: "una negociación constructiva que involucra al menos dos, y usualmente más, sujetos conscientes y políticamente significativos" (Clifordd, 1988: 159); un diálogo negociativo entre las necesidades auténticas de las cantoras y del pueblo afroserrano en general, con las necesidades propias de quién lleva el proyecto. Si tanto las necesidades de la gente como las del investigador/a no son respetadas, en el primer caso, se eliminaría la participación y los efectos positivos en la gente y, en el segundo caso, se mataría la fuerza interior que permite llevar un proyecto con pocos recursos y sin ninguna remuneración económica. De esta manera, el proyecto, el cual inicio con la idea de un pequeño folleto- $C D$, al encontrarse con una cultura que ha caminado durante siglos sobre el manto de la oralidad, ${ }^{1}$ debió utilizar el mismo lenguaje y adaptarse también al lenguaje de la modernidad: la tecnología. Es de esta manera que nace el documental "Las Tres Horas y Cantos de Difuntos. Ritualidad del pueblo afroserrano del Ecuador".

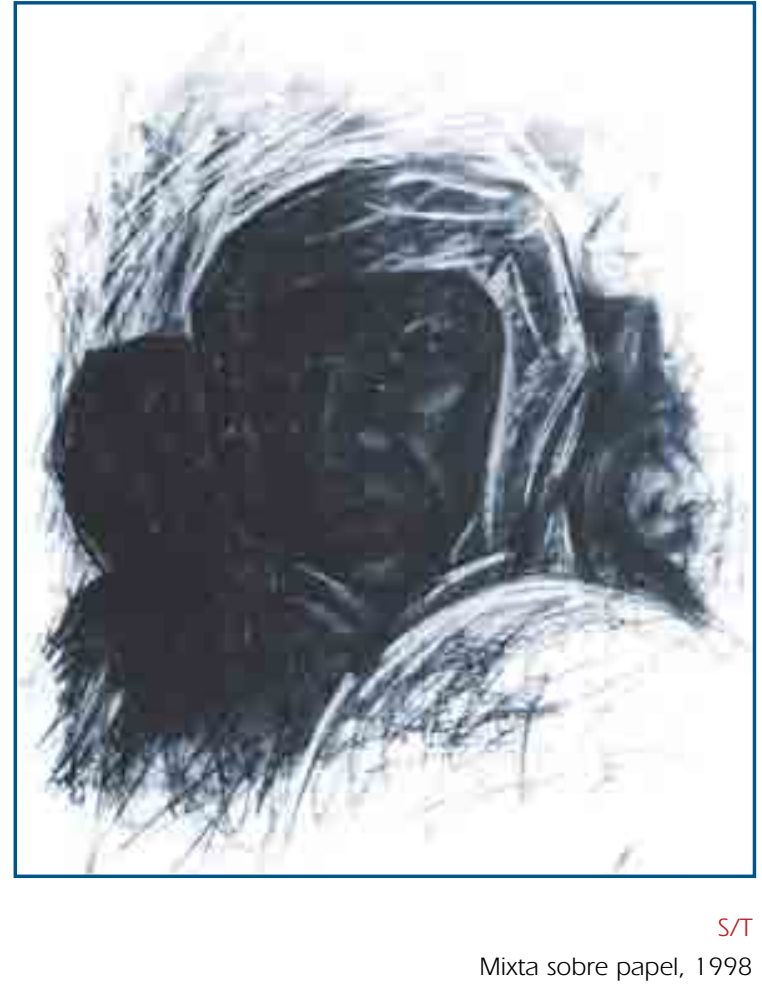

En las conversaciones mantenidas con los diferentes grupos de cantoras me di cuenta que las necesidades de los grupos que viven en las comunidades y las de quienes residen en las ciudades son diferentes. Como lo mencioné al inicio, la idea de realizar un evento en donde se socialice y se visibilicen las tradiciones del pueblo afroserrano nació en el grupo de cantoras residentes en Quito. Ellas mencionaban que les gustaría invitar a sus jefes, quienes para algunas de ellas, son los dueños de la casa en donde trabajan como empleadas domésticas. Sentí y concluí, entonces, que existe una necesidad por parte de la población afroserrana, residente en las ciudades, de ser visibilizadas para ganarse el respeto del medio en el que se desenvuelven, dentro de un espacio donde la alteridad es su

1 En el curso "La diáspora Afro andina” dado por la Universidad de Duke en convenio con la Universidad Politécnica Salesiana (MarzoJunio 2011 ), el profesor Juan García reiteró varias veces esto. 


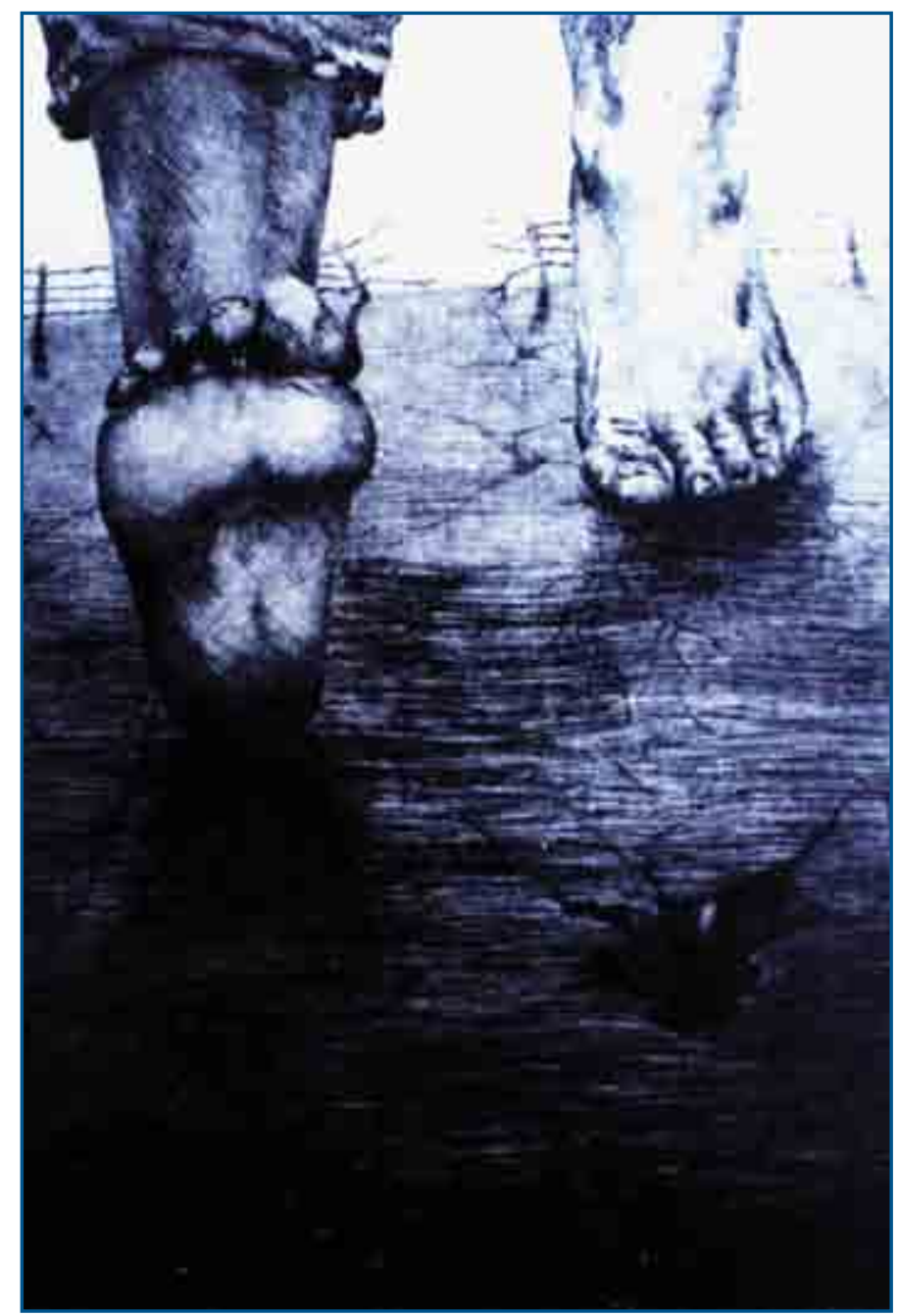

Siempre p'lante Grabado, 1994 realidad y la homogenización es su objetivo: como dice el proverbio "no se puede amar y respetar a lo que no se conoce".

En la ejecución del proyecto, al encontrarme frente a herramientas y situaciones que nunca había manejado, tales como las requeridas para la producción del video (cámaras, sonido, luz, guión, etcétera), para la elaboración del texto, del $C D$ y en general de todo el material que iba surgiendo en el proyecto, tuve dos opciones: la primera, esperar a tener mayores posibilidades económicas y capacidades cognoscitivas para lograr hacer un producto 'perfecto', y la segunda, arriesgarme con lo que tenía en ese momento, apuntando, más que a la perfección, a la autenticidad, espontaneidad y utilidad, tanto para un proceso de autoreconocimiento (Martínez, 2008) como para su visibilización. Creo que por mi temperamento y por la necesidad que percibía en la gente escogí la segunda opción. Una de estas situaciones desconocidas la encontré al darme cuenta de la subjetividad con la que se estaba desarrollando el documental. El peso y la carga que esto representaba se aligeraron cuando comprendí que, frente a la subjetividad, lo que hace la diferencia entre el respeto y el irrespeto hacia los seres humanos, es cuan cercano física y emocionalmente está uno a la gente.

Desde un inicio tuve la intención de que el proyecto llegue, tanto al pueblo afroserrano como a otros sectores de todas las edades. Es así que, al momento de sentarme a escribir se me presentaron grandes dificultades: ¿cómo escribir en un lenguaje sencillo sin perder el ojo académico?2 ${ }^{2}$ No fue fácil y no estoy segura de haberlo logrado. Si bien el texto es pequeño, tenía muy claro lo que quería mostrar en él: quería invitar al lector/a a acercarse a la cultura del pueblo afroserrano, a vivir sus rituales, en donde los cantos -un elemento imprescindible de su reli-

2 Algunas discusiones referentes al tema de la escritora etnográfica se les puede encontrar en Clifford (1988), Marcus y Cushman (1982) y Leyotard (2004). 
giosidad- son ejecutados. Quería visibilizar una historia que muy pocos conocen; no la historia oficial del Ecuador y de América entre españoles e indígenas; sino quería contar una historia en donde se evidencie que los negros y las negras esclavizados/as ${ }^{3}$ han sido una fuerza fundamental para el desarrollo industrial (2008: 59) de los países del 'primer mundo', y para las conquistas y formaciones del 'tercer mundo' (2008: 5). No obstante -incluso en la estructuración del textoya existía controversia entre la sencillez y lo académico. Para el mundo académico, generalmente se va parte de lo macro, desde el contexto general (histórico, social, político) para ir hacia lo micro: los cantos. Desde mi sentir, la historia debe dejar de ser un espectáculo en donde los lectores son solamente espectadores de los acontecimientos: la historia es una invitación a la autoreflexión (de reflejo) desde el presente hacia el pasado. Es así que inicié el texto describiendo los escenarios de acción en donde los cantos son ejecutados.

\section{Resultado de la investigación Los Cantos, fuentes, raíces y sentimientos}

El canto de Las Tres Horas y los Cantos de Difuntos, cantados dentro de esta área geográfica, presentan variaciones significativas que los hacen únicos debido a sus particularidades culturales e históricas. Es así que tenemos claramente tres influencias decisivas: la española colonizadora, la raíz africana y la presencia indígena local.

El canto de Las Tres Horas, cantado en Semana Santa luego de cada una de las siete palabras que dijo Jesucristo antes de morir, las cuales son predicadas por el sacerdote, es una tradición católica que ha sido adaptada a los timbres y colores de las voces del pueblo afroserrano, mostrando melodías propias en cada comunidad. De esta manera es probable que su sentido no sea el mismo que el de la religión católica.

En cuanto al Grito de las Almas, cantado por el Animero en el ritual de Saque de las Almas en el mes de octubre, junto con las Salves, cantadas en los velorios de las personas adultas, también presentan diferencias melódicas de una comunidad a otra. Al parecer no existe una fuente común de estos cantos: algunas vienen de la Iglesia española del cancionero católico nacional, otras tienen una raíz en la Iglesia universal, ya que son conocidas también en algunas comunidades indígenas o mestizas (Peters et al., 2005).

La Salve Regina, por ejemplo, ha sido identificada hasta el momento como la primera canción dedicada a la Virgen María. Fue compuesta por el obispo San Pedro de Mezonzo (930-1003), como un pedido de socorro y protección a la Virgen frente al ataque de Abi Amir Muhammad a un asentamiento cristiano. ${ }^{4}$ Cuando llega la Salve Regina a la Audiencia de Quito, en una sesión del Cabildo Catedralicio celebrado en 1579, se le exige a Diego Lobato, músico de las cátedras, ejecutar la Salve cada domingo y en todas las fiestas y días de predicación (Stevenson, 1989: 15). En 1580, Francisco de Zúñiga, secretario de la Real Audiencia de Quito, donó cien pesos a la Iglesia, con la condición de que la Salve Regina fuera cantada todos los sábados por los músicos de la catedral y acompañados por el órgano (1989:16).

Por su parte, el musicólogo español, Ismael Fernández de la Cuesta menciona que las Salves fueron cantadas en los pueblos después de haber sido traducidas al castellano, ya que originalmente fueron escritas en latín. De igual manera, dice que mantienen relación con los

3 En el curso "La Diáspora Afro andina" (Marzo-Junio 2011 ) se menciona la importancia de comenzar a utilizar el término de esclavizados y no esclavos, ya que este segundo término 'naturaliza' la situación de esclavitud.

4 <http://sagradafamilia.devigo.net/santoral/septiembre/10septiembre.htm>. 
cantos gregorianos, impuestos en toda España en el siglo XII. ${ }^{5}$

Otra de las raíces de los cantos rituales del pueblo afroserrano ha sido encontrada en la poesía española colonial de los romances, pero siempre combinados con la manera africana de cantar (2005), tanto por la forma de diálogo como por el 'color', y con los 'glissandos' que consiste en resbalar de un sonido (nota musical) a otro, característicos en la música africana y andina.

Dentro de este panorama africano podemos hablar de los 'griots', que pueden tener algo que ver con los cantos actuales del pueblo afroandino. Estos personajes han existido desde tiempos inmemorables; eran personas dedicadas a transmitir acontecimientos históricos a través del canto 'a capela', esto es, sin más instrumento que la voz, deambulando de un lado a otro, al igual que lo hacían los juglares o los trovadores españoles (Gutierrez, 2000). En el mismo continente africano encontramos a las mujeres antiguas de Loango-Angola, que lloraban y entonaban en los rituales llamados "cantos de la casa de los muertos" o "cantos de lágrimas" (Estudio del Acontecer Afrocolombiano, 1996). Esto nos acerca a una de las principales características de las religiones animistas africanas: el culto a los antepasados (Delumeau, 1997).

En este sentido, para la misionera Federica Peters (2005), en el caso tanto de los africanos como de los afroamericanos, la religión católica cristiana se ha insertado en las bases de las religiones africanas, en donde los elementos católicos son entendidos desde la forma africana, lo que quiere decir para la autora que Jesús, Dios, los Santos (as) y los antepasados tienen una presencia actual y real en la vida y en las comunidades del pueblo afroandino. Así, cuan- do se llora por la muerte de Jesús en Semana Santa, por quien se está llorando es por algún ser querido y por los antepasados. Esta presencia de los antepasados en las comunidades afroandinas se evidencia en el Saque de las Almas, rito en el cual las almas visitan y se pasean por las comunidades; las ánimas benditas necesitan volver al mundo que dejaron atrás, porque ellas siguen siendo parte de la comunidad (Chalá s/f).

Cabe mencionar que para el antropólogo español Idelfonso Gutiérrez Azopardo (2000), los ritos africanos que adquirieron una mayor preponderancia en América fueron los ritos funerarios, en donde se encuentran rasgos africanos muy acentuados. Frente a esto, la etnóloga mexicana Luz María Martínez (2008) piensa que en los rituales fúnebres, lo que se canta, no es del todo católico sino que son cantos que ayudan a dirigir al alma del difunto hacia su morada definitiva.

En cuanto a la influencia indígena es importante remitirnos a la historia de la fundación de la ciudad de Ibarra. Se cuenta que la nieta de Atahualpa fue quien donó los terrenos para la fundación de la nueva ciudad que se fue poblando con familias españolas-indias. Por eso, en la interrelación con el mundo indígena, el pueblo afroserrano ha adquirido varias formas de la lengua kichwa, como la introducción de la 'ka' en medio de una oración: "yo ka no voy"; "ya ka la señora dijo"; o el uso de la vocal 'i' en vez de la 'e': "sabimos", "habimos", "hacimos", como se la emplea en el kichwa. ${ }^{6}$

En los cantos, la influencia indígena se la percibe también en los 'glissandos' y en las melodías en donde se encuentran la pentafonía (cinco notas o sonidos) andina. Un ejemplo de esto encontramos en la Salve "Salve, Salve, Gran Señora”, la cual tiene una melodía de Yaraví. 


\section{A modo de conclusiones}

Los aprendizajes que dejan una experiencia dentro de un proyecto de investigación son muchísimos; algunos de ellos ya hemos compartido a lo largo del presente trabajo: intuiciones que se reafirman y se confirman, otras que se descartan y se transforman.

La cantora Jael García del grupo de Chalguayacu me cuenta que en esta última Semana Santa, el canto de Las Tres Horas salió 'bien bonito' y ahora hay más jóvenes que quieren unirse al grupo. También, las cantoras han podido verse a través del video en sus hogares, cerca de sus hijos y nietos. Luego del lanzamiento, mucha gente del público mencionó que nunca había escuchado esos cantos y que no sabía que el pueblo afroserrano (algunos ni sabían que existía) realizaba estas prácticas rituales. Estas experiencias, junto a muchas otras, me confirman que el proyecto ha tendido un gran alcance.

Sin embargo, me queda la sensación de que la participación por parte de las cantoras, $\mathrm{y}$ en general de la gente, hubiera podido ser mayor. En el video, por ejemplo, hubieran podido ser ellos y ellas quienes elaboraran el guión; o tal vez, los jóvenes de las comunidades podrían haberse interesado en llevar las cámaras, los micrófono, etcétera. Tal y como se lo está planteando en el Colectivo de Producción Afroecuatoriana, conformado en el mes de mayo por personas afroecuatorianas provenientes de diferentes provincias.

Siento que se debe apuntar más a lo que Juan García, quien desde hace ya varios años ha trabajado en la implementación de nuevos métodos educativos que permitan fortalecer la identidad de los niños, niñas y jóvenes afroecuatorianos plantea: la educación casa adentro "para fortalecer lo propio de lo que nos hablan los ancestros [...] un proceso de permanente reflexión y construcción colectiva, mediante el cual se fortalece la identidad del pueblo que

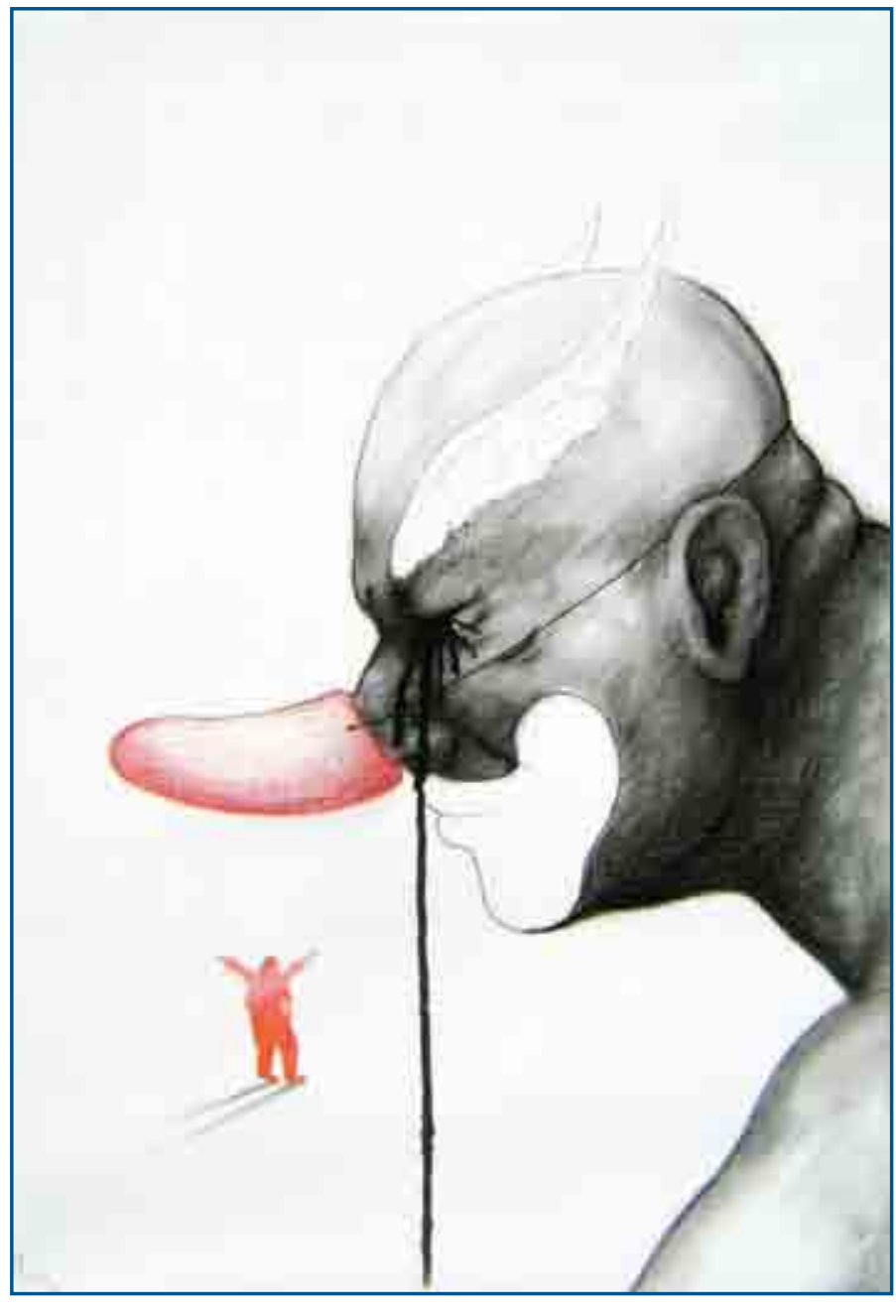

De la serie Fractales 
la asume" (García s/f): "Primero tenemos que llenar el corazón con lo que es propio, para lo ajeno llegue pero no pueda entrar y si entra, no tenga un lugar donde quedarse. ${ }^{7}$

\section{Bibliografía}

- Ayala Santos, Ana Gilma. Amuletos y Santos en el Atrato. Medellín: Ed. Mundo Libre. 2000.

- Chalá, María Elena (Comp.) (s/f) Etnoeducación afro ecuatoriana. Serie: Historia de Vida: Don Perfilio Lara.

- Clifford, James. The Predicament of Culture. Cambridge: Harvard University, en: Reynoso, Carlos (1991). El Surgimiento de la Antropología Posmoderna. México: Ed. Gedisa, 1988.

- Delumeau, Jean (Dir.). El hecho religioso, Una enciclopedia de las religiones de hoy. Madrid: Siglo XXI, 1997.

- Estudio Acontecer Afrocolombiano. El Alabao, cantos fúnebres de la tradición oral del pacifico colombiano. Valle del Cauca. Biblioteca Departamental, 1996.

- García, Juan. Tradición Oral, una herramienta para la etnoeducación. Esmeraldas, Ecuador (s/f).

- Guerrón, Carla. El color de la Panela. Quito: Ediciones Afroamérica, Centro Cultural AfroEcuatoriano, 2000.

- Guerrero C., Fernando. Dinámica Poblacional y Estructura Agraria en algunas comunidades de la cuenca del río Mira y el valle del Chota. Ecuador, Conferencia Episcopal Ecuatoriana, 1996.

- Gutiérrez Azopardo, Ildefonso. La población negra en América. Bogotá: El Búho, 2000.

- IFA, Instituto de formación afroecuatoriana. Talleres de identidad. Quito. Pastoral afro de Quito, 2007.

- Lyotard, Jean-François. La condición posmoderna, informe sobre el saber. Madrid: Ed. Cátedra, 2004.
- Marcus, E., George y Dicke E. Cushman. Annual Review of Anthropology, vol. II en Reynoso, Carlos (1991): El Surgimiento de la Antropología Posmoderna. México: Ed Gedisa, 1982.

- Martínez Montiel, Luz María. Africanos en América. La Habana: Editorial Ciencias Sociales, 2008.

- Mullo, Juan. Los Cantos de Las Salves y Las Cantoras, una manifestación de un canto social $y$ sagrado del pueblo afro-andino. Quito: Banco Central del Ecuador, 2008.

- Pabón, Iván. Identidad Afro. Quito: Abya-Yala, 2007.

- Pareja Diezcanseco, Alfredo. Historia del Ecuador. Quito: Ed. Colón, 1962.

- Peters, Federica. et al. Sobre-vivir a la propia muerte. Quito: Abya-Yala, 2005.

- Puente Ojea, Gonzalo e Ignacio Careagua Villalonga. Animismo, el umbral de la Religiosidad. Madrid: Ed. Siglo XXI, 2005.

- Rua, Juan Guillermo. Vida y Muerte en el litoral; en Lucía Ortiz (Ed.): Chambacú, la historia la escribes $t u$, Ensayos sobre cultura afrocolombiana. Editorial Iberoamericana, 2007.

- Savoia, P. Rafael y Javier Gomezjurado (recop), "El Negro en la historia del Ecuador". Conferencias del Cuarto Congreso de Historia del Negro en el Ecuador, Centro cultural afroecuatoriano, Ediciones Afroamérica, 1999.

- Stevenson, Robert. La música en Quito. Quito: Banco Central del Ecuador, 1989.

- Tuner W., Victor. Los símbolos en el ritual Ndembu en Marco Rueda y Segundo Moreno (comp): Cosmos, Hombre y Sacralidad. Quito: Abya-Yala, 1997.

\section{Referencias electrónicas}

- <http://sagradafamilia.devigo.net/santoral/ septiembre/10septiembre.htm>.

7 Esta frase la dicen los mayores del pueblo afroesmeradeño. Fue tomada del libro La Tradición Oral: una herramienta para la etnoeducación de Juan García. 